\title{
BSA-Seq and Genetic Mapping Reveals AhRt2 as a Candidate Gene Responsible for Red Testa of Peanut
}

Kun Zhang

Shandong Academy of Agricultural Sciences

Mei Yuan

Shandong Academy of Agricultural Sciences

Han Xia

Shandong Academy of Agricultural Sciences

Liangqiong $\mathrm{He}$

Guangxi Academy of Agricultural Science

Jing Ma

Shandong Academy of Agricultural Sciences

Mingxiao Wang

Shandong Academy of Agricultural Sciences

Huiling Zhao

Shandong Academy of Agricultural Sciences

Lei Hou

Shandong Academy of Agricultural Sciences

Shuzhen Zhao

Shandong Academy of Agricultural Sciences

Pengcheng Li

Shandong Academy of Agricultural Sciences

Ruizheng Tian

Shandong Academy of Agricultural Sciences

Jiaowen Pan

Shandong Academy of Agricultural Sciences

Guanghui Li

Shandong Academy of Agricultural Sciences

Mahendar Thudi

Shandong Academy of Agricultural Sciences

Changle Ma

Shandong Normal University

Xingjun Wang 
Shandong Academy of Agricultural Sciences

Chuanzhi Zhao ( $\square$ chuanzhiz@126.com )

Shandong Academy of Agricultural Sciences https://orcid.org/0000-0001-7465-7425

\section{Research Article}

Keywords: Peanut, Red testa, BSA-Seq, AhRt2, Diagnostic marker

Posted Date: December 6th, 2021

DOI: https://doi.org/10.21203/rs.3.rs-1109892/v1

License: (c) (1) This work is licensed under a Creative Commons Attribution 4.0 International License. Read Full License

Version of Record: A version of this preprint was published at Theoretical and Applied Genetics on February 15th, 2022. See the published version at https://doi.org/10.1007/s00122-022-04051-w. 


\section{Abstract}

Testa color is an important trait of peanut (Arachis hypogaea L.). Peanuts with red testa are rich in anthocyanin, are very popular with consumers. However, genes responsible for the red testa trait in peanut are rarely reported. In order to fine map red testa gene, two $\mathrm{F}_{4}$ populations were constructed through the cross of YZ9102 (pink testa) with ZH12 (red testa) and Zhanhong2 (red testa). Genetic analysis indicated that red testa was controlled by a single recessive gene, and named as AhRt2 (Red testa gene 2). Using BSA-seq approach, AhRt2 was preliminary identified in chromosome 12, and further mapped to a 530-kb interval using 220 recombinant lines through linkage mapping. Functional annotation, expression profiling, and sequence variation analyses confirmed that the anthocyanin reductase (ANR), Arahy.IK60LM, was the most likely candidate gene for AhRt2. A SNP in the third exon of AhRt2 changed the encoding amino acids, was associated with red testa of peanut. In addition, a closely linkaged molecular marker to red testa trait was developed. Our result provide insight into the molecular mechanism underlying peanut testa color and provide valuable diagnostic marker for marker-assisted selected (MAS) breeding in peanut.

\section{Key Message}

The candidate recessive gene AhRt2 responsible for red testa of peanut was identified thorugh combined BSA-seq and linkage mapping approaches.

\section{Introduction}

Peanut (Arachis hypogaea L.) is widely grown in more than 100 countries, with a total production of approximately 48.8 million tons in 2019 (http://www.fao.org/faostat/en/\#data/QC). Peanut is known as an important cash crop not only for serving as high quality cooking oil but also for a variety of snacks. Peanut is superior in nutrition because it is enriched in many nutritional ingredients, such as vitamin B1, B3, B9 and E, biotin, resveratrol, isoflavones, phytic acid, anthocyanin and procyanidins, and many of which are accumulated in the testa (seed coat) (Pandey et al., 2012, Zhao et al., 2012, Zhao et al., 2020).

The testa color is an important trait of peanut and with a lot of variations, such as white, pink, red and black (or deep purple). The majority of peanut varieties have pink testa. Anthocyanin content and composition are important factors to determine the color of testa. In higher plants, there are six kinds of anthocyanins including delphinidin, cyanidin, pelargonidin, peonidin, petunidin and malvidin. Significant differences were found for the types and content of anthocyanidin in different testa color peanuts, and the content of delphinidin, cyanidin and pelargonidin were closely related to the testa color (Li et al., 2017). Recently, the metabolome comparison results suggested that the accumulation of petunidin and cyanidin was higher in red testa than that in the pink testa of peanut (Xue et al., 2021). Anthocyanins have strong antioxidant capacity and important nutritional value (Shin et al., 2006, Winkel-Shirley, 2001). High-anthocyanin varieties have become one of the important directions of rice and wheat breeding (Giordano et al., 2017, Ito \& Lacerda, 2019). The testa was developed from the integument and with the 
same genotype as its maternal plant, thus, the phenotype of testa color appears one generation late than other traits (Chen et al., 2021, Zhao et al., 2020). For traditional breeding methods, the phenotype of the testa color can only be identified after harvest, which prolonged the screening time. Marker-assisted selection (MAS), selecting the target progenies according to the genotype, can significantly accelerate breeding process and improve breeding efficiency, especially for testa color selection. For MAS approach, revealing the genetics and developing DNA markers tightly linked the traits are required.

With the rapid development of next-generation sequencing (NGS), considerable progress has been made in peanut whole genome sequencing including both wild type and cultivated peanuts (Bertioli et al., 2016, Bertioli et al., 2019, Chen et al., 2019, Yin et al., 2018, Zhuang et al., 2019). The availability of the completed genome sequencing of peanuts provided ideal resource for genome-wide identification of SSR and SNP markers in silico (Zhao et al., 2017, Ma et al., 2020). In addition, large-scale 58K SNP Array (Axiom_Arachis, v1) and 48K SNP Array (Axiom_Arachis2, v2) have been developed, which provide a new chance for genetic analysis, constructing high-resolution linkage maps, and QTL mapping (Clevenger et al., 2017, Clevenger et al., 2018b, Nabi et al., 2021). Moreover, great efforts have been made in the genetic map construction, fine mapping, and MAS of peanut (Agarwal et al., 2018, Han et al., 2018). The major gene related to the black testa color of peanut have been identified through BSA-seq and eQTL approaches (Zhao et al., 2020, Huang et al., 2020).

The previous studies suggested that peanut the red testa was controlled by one dominant gene $(R 1)$ and two recessive genes $(r 2, r 3)$, and all these three genes appeared to inherit independently (Branch, 2011). Recently, a major dominant gene controlling red testa color, AhRt1, was fine-mapped on chromosome A03, and provided the closely linked makers for MAS breeding (Chen et al., 2021, Zhuang et al., 2019). However, the recessive gene in controlling the red testa color was poorly characterized.

In the present study, one recessive gene controlling red testa, AhRt2, was fine-mapped to a $0.5 \mathrm{Mb}$ genomic region in chromosome 12 using the BSA-seq and linkage mapping approaches. An anthocyanidin reductase (ANR) gene was suggested to be the possible candidate gene. A "G/A" SNP is in the third exon of the ANR gene. We also developed tightly linked molecular markers which could be used in future MAS breeding.

\section{Materials And Methods}

\section{Development of mapping population}

For inheritance analysis and constructing the mapping populations, the pink testa peanut cultivar Yuanza 9102 (YZ9102) was used as the female parent to cross with the red testa peanut cultivars Zhonghua 12 (ZH12) and Zhanhong 2 (ZHH2), respectively (Table 1). Two populations (YZZH12 and YZZH2) were developed through single-seed-descent method. The $F_{1}, F_{2}$ and $F_{3}$ plants of two populations were planted in the field from 2017 to 2019 at Laixi Experimental Station $₫ 36^{\circ} 48^{\prime} 47.41^{\prime \prime} \mathrm{N} \unrhd 120^{\circ} 30^{\prime} 22.54 " \mathrm{E} \otimes$, Shandong Peanut Research Institute (SPRI), Shandong, China. The $F_{4}$ and $F_{5}$ plants were planted in the field from 
2020 to 2021 at Jiyang Experimental Station of Shandong Academy of Agricultural Sciences (SAAS), Shandong, China $\varangle 36^{\circ} 58^{\prime} 34.53^{\prime \prime} \mathrm{N} \otimes 116^{\circ} 59^{\prime} 1.29 " \mathrm{E}$.

\section{Measurement of anthocyanin}

The anthocyanin content of peanut testa was measured using improved method as previous reports (Mancinelli et al., 1991, Teng et al., 2005, Zhao et al., 2020), using at least three sets of more than eight seeds per sample. In brief, frozen peanut testa (approximately $50 \mathrm{mg}$ ) was ground in a $5 \mathrm{~mL}$ centrifuge tube using liquid nitrogen. Then, homogenized testa was extracted at $4^{\circ} \mathrm{C}$ by adding $700 \mu \mathrm{l}$ acidic methanol (the volume ratio of methanol to $\mathrm{HCl}$ is 99:1). After overnight incubation, the homogenates were centrifuged for $1 \mathrm{~min}$ at $12000 \mathrm{rpm}$ for $10 \mathrm{~min}$. The supernatant (approximately 600 $\mu \mathrm{l})$ was collected and mixed with $1 \mathrm{~mL}$ trichloromethane and $400 \mu$ listilled water, and centrifuged at $4^{\circ} \mathrm{C}$ at $12000 \mathrm{rpm}$ for $10 \mathrm{~min}$. Then, the absorbance of the supernatant was measured in spectrophotometer (U-3000, HITACHI, Japan) at 530 and $657 \mathrm{~nm}$, respectively. The relative anthocyanin content was calculated according to the absorbance with the formula of $\left[A_{530}-\left(1 / 4 \times A_{657}\right)\right]$ and then normalized by sample weight.

\section{Whole genome sequencing and BSA-seq analysis}

The genomic DNA was extracted from the leaves using the DNA Extraction Kit (DP305) of TIANGEN Biotech (Beijing,China) according to the manufacturer's instructions. DNA quality was determined using the BioPhotometer plus spectrophotometer (Eppendorf AG, Hamburg, Germany) and 1\% agarose gel electrophoresis. For BSA-seq, two DNA pools were constructed by mixing equal amounts of DNA from 30 red testa $\mathrm{F}_{2: 4}$ individuals (Red-pool) and 30 pink testa $\mathrm{F}_{2: 4}$ individuals (Pink-pool). Then, DNA of YZ9102 and $\mathrm{ZH} 12$, and two DNA pools were sequenced on BGISEQ-500 platform at the Beijing Genomics Institute (BGI).

After sequencing, clean reads were obtained by removing low-quality and short reads using Soapnuke program (Chen et al., 2018), and mapped on reference genome of cultivated peanut Tifrunner (https://peanutbase.org/peanut_genome) using BWA software with the SAM tools (Li \& Durbin, 2009). Single-nucleotide polymorphism (SNP) and Insertion/Deletion(InDel) were called, and filtrated by removing heterozygous and missing SNPs and InDels in the pools and parental lines using GATK software (McKenna et al., 2010). The SNP-index represents the ratio of reads harboring SNPs among the entire number of reads (Abe et al., 2012). To identify candidate regions associated with the red testa trait, the $\Delta$ SNP-index of each locus was calculated by subtracting the SNP-index of the Pink-pool from that of the Red-pool according to previous method (Takagi et al., 2013). To confirm the results of $\Delta$ SNP-index, Euclidean Distance (ED) algorithm was further preformed to identify the SNPs and InDels associated with the red testa trait using the equation reported previously (Lei et al., 2020, Hill et al., 2013).

\section{Marker development, genetic map construction and mapping}


To validate the BSA-seq results and further narrow down the region, $21 \mathrm{InDels}$ in the candidate region were selected according to the comparative genomic information among the parents. Twenty one primer pairs were designed to the flanking sequences of the tragetted SNPs using Primer Premier 5.0 (http://www.premierbiosoft.com/primerdesign/). The polymorphism of these InDels was confirmed through polyacrylamide gel electrophoresis as described previously (Zhao et al., 2017). The Indel markers showing polymorphism among the parents were further used to genotype the $F_{2: 4}$ population. The sequences of primers used for mapping are listed in Supplementary Table S1.

Genetic linkage map was constructed using JoinMap 5.0 software (https://www.kyazma.nl/index.php/JoinMap/). The recombinant ratio was converted into genetic distances (centimorgans, $\mathrm{cM}$ ) through the function of Kosambi map. The linkage groups were calculated at a minimum LOD score of 5. MapChart 2.3 software was used for drawing the linkage maps (Voorrips, 2002). For QTLs analysis, inclusive composite interval mapping of additive (ICIM-ADD) was performed using software QTL IciMapping V4.1 (Meng et al., 2015). The LOD threshold was calculated by 1,000 permutations at $\mathrm{P}<0.05$, and the LOD score was set at 2.5 to determine the presence of a putative QTL associated with a target trait.

\section{Prediction of candidate genes}

The sequences of gene information in the candidate interval were obtained according to the cultivated peanut reference genome sequences (Version 1, https://peanutbase.org). The functions of candidate genes were annotated through Blastx program in databases of $\mathrm{Nr}$ (NCBI, http://www.ncbi.nlm.nih.gov), GO (https://www.geneontology.org/), KOG (http://www.ncbi.nlm.nih.gov/KOG), and KEGG (http://www.genome.jp/kegg). For sequence alignment and phylogenetic analysis of the anthocyanidin reductase (ANR) genes, the amino acid sequences of ANR proteins were download from NCBI. Multiple sequence alignments were performed by clustalw (https://www.genome.jp/tools-bin/clustalw) and BoxShade online program (https://embnet.vital-it.ch/software/BOX_form.html). The phylogenetic analysis was produced by MEGA7 (https://www.megasoftware.net/) with the neighbor-joining statistical method. The GenBank accession numbers of these proteins are provided in Supplementary Table S2.

\section{Quantitative Real-Time PCR (qRT-PCR)}

Based on Functional annotation of genes, 14 genes were selected for qRT-PCR analysis. After harvest from field, the seed coat was collected immediately before drying. The samples for qRT-PCR analysis are the same as that for BSA-seq, including two parents and RNA pools, and three biological replicates were prepared for every sample. Total RNA was extracted using Trizol Reagent kit (TaKaRa, Inc., Dalian, China) according to the instructions of manufacturer. The reverse transcriptions were performed with PrimeScript II 1st Strand cDNA Synthesis Kit (TaKaRa, Inc., Dalian, China). The primers for qRT-PCR were designed using Primer Premier 5.0 (http://www.premierbiosoft.com/primerdesign/) and listed in Supplementary Table S1. The qRT-PCR reactions were performed on ABI7500 Real Time System (USA) as 
previous studies (Wang et al., 2017). The relative expressional levels of genes were calculated by $2^{-} \triangle \Delta C T$ method.

\section{Results}

\section{Phenotyping and Genetic Analysis of Red Testa in Peanut}

The phenotypic analyses showed significant differences in testa color between the two parents. For YZ9102, the testa color is traditionally pink, while ZH12 is red in testa color (Figure 1A). The red testa has more anthocyanin than that in pink one (Figure 1B). The total average content of anthocyanin in the red testa lines is about 2-7 times higher than that in the pink lines.

In order to gain insights into the genetic inheritance of red testa in peanut, the pink testa YZ9102 was crossed with the red testa Zhonghua12 ( $\mathrm{ZH} 12)$ and Zhanhong2 (ZH2) and two segregating populations, YZZH12 and YZZH2, were developed respectively (Table 1). All $F_{1}$ and $F_{2}$ seeds showed pink testa, and the $F_{3}$ seeds displayed different testa color corresponding to the coloration of either $Y Z 9102$ or $\mathrm{ZH} 12 / \mathrm{ZH} 2$. Among the $220 \mathrm{~F}_{4}$ individuals of $\mathrm{YZZH1} 12$ population, 143 exhibited pink testa, and 77 showed red testa, corresponding to a segregation ratio of 5:3 by the Chi-square test $\left(\chi^{2}=0.59, p=0.05\right)$. Similarly, $Y Z Z H 2$ population, $F_{4}$ individuals also showed a $5: 3$ segregation ratio of pink and red $\left(\chi^{2}=0.69\right.$, $p=0.05$ ) (Table 1). These results demonstrated that the red testa of peanut should be controlled by a single recessive gene, and named as AhRt2.

\section{BSA-seq Analysis and Mapping of Gene AhRt2}

To map the AhRt2 gene, we constructed two extreme pools, pink-pool and red-pool, which comprised 30 pink and 30 red $\mathrm{F}_{2: 4}$ plants, respectively. The two parents and two extreme pools were sequenced using the BGISEQ-500 platform. In total, $31.57,41.22,113.25$ and $120.38 \mathrm{~Gb}$ raw data were generated for the YZ9102, ZH12, Pink-pool and Red-pool, representing approximately 12.34x, 16.12x, 47.08x and 44.29x genome coverage, respectively (Table 2). The filtered clean reads of each sample were mapped to the reference genome of the cultivar Tifrunner and a total of 412,874 SNPs/InDels were identified. To obtain the genomic region associated with the red testa, two approaches, Delta SNP and ED algorithms, were performed to calculate the allele segregation of the SNPs and InDels between the two extreme DNA pools. Delta SNP and ED algorithms showed that $54.56 \%$ and $70.11 \%$ candidate SNPs/InDels enriched on chromosome 12 (Chr.12), respectively (Figure 2A and Figure 2B). On Chr.12, the $7.8 \mathrm{Mb}$ region (109.9Mb$117.7 \mathrm{M})$ exhibiting significant linkage disequilibrium was identified as the candidate region for red testa (Figure 2C).

\section{Fine Mapping of the AhRt2 Gene}

According to the BSA-seq results, $21 \mathrm{InDel}$ markers were developed in the candidate region of Chr.12, and 11 of them displayed stable polymorphisms between the parental line and the $F_{2}$ individuals. These 
markers were used for constructing the genetic map of candidate region and QTL mapping using the 220 $\mathrm{F}_{2: 4}$ individuals of $\mathrm{YZZH} 12$ population. The total length of the linkage map is $59.27 \mathrm{cM}$, which is corresponding to $110.05 \mathrm{Mb}$ to $177.62 \mathrm{Mb}$ region of chr.12. The major genewas mapped in a $3.64 \mathrm{cM}$ region between Indel marker InDel_16 and InDel_20 (Figure 3). The physical location of AhRt2 was narrowed in a $0.53 \mathrm{Mb}$ region (Chr12: 117.03Mb-117.56Mb)(Figure 3).

\section{Identification of the Candidate Genes Related to Red Testa}

There are 52 genes in the candidate $0.53 \mathrm{Mb}$ region of Chr.12. Among them, two genes (Arahy.JFV18T and Arahy.X3FWT9) were annotated as bHLH transcriptional factor genes, one of the core members to form the MYB-bHLH-WD40 (MBW) complex, and the latter is regarded as an important regulatory gene in plant anthocyanin biosynthesis. In addition, the candidate interval contains one anthocyanidin reductase (ANR) gene (Arahy.IK60LM), one of the structural genes in anthocyanin synthesis pathway, and named as AhANR1 (Table 3). Resequencing results showed that there are 36 SNPs and 7 InDels in the candidate interval. Most of these SNPs/InDels were located in the intergenic region, and 9 SNPs were located in the gene region including two SNPs in the AhANR1 gene region (Table 3). One SNP "SNP_11719149" was located in the $-312 \mathrm{bp}$ of the upstream of the Coding DNA Sequence (CDS). The other SNP "SNP_117190528" was located in the third exon of the ANR gene with a $\mathrm{C} / \mathrm{T}$ variation, which leads to a transition from Thr (AㄷT) in YZ9102 to lle (AIT) in ZH12(Figure 4A).

\section{Sequence Alignment and Expression analysis of the Candidate Genes}

AhANR1 of YZ9102had the same sequence with A. duranensis and A. ipanesis, Tifrunner, Shitouqi and Fuhuasheng, which the whole genome sequences have been released. Sequence alignment showed that threonine is conserved both in A. duranensis and A. ipanesis and other plant species, such as rice (Oryza sativa), common bean (Phaseolus vulgaris), soybean (Glycine max), and tobacco (Nicotiana tabacum) (Figure 4B). To further analyze the candidate region, 13 genes were selected for qRT-PCR assay. Interestingly, we found that the expression of the ANR gene was up-regulated in both the red testa ZH12 and the red testa pools(Figure 4C). qRT-PCR showed that the CT Means of the two bHLH genes (Arahy.JFV18T and Arahy.X3FWT9) exceeded to 35, indicating that the expression level of these two bHLH genes is very low in both red and pink testa. We also detected the expression level of the candidate gene of AhRt1 in YZ9102 and ZH12, and found that the expression of AhRt1 is very low with the Ct mean $>35$. So, these two bHLH genes and the candidate gene of AhRt1 were not likely the genes responsible for the red testa color in population used in this study. Taken together, we predicted that the AhANR1 might be the key gene controlling the red testa.

\section{Discussion}

\section{BSAseq is an effective strategy for gene fine mapping}


BSA-seq approach is a rapid method for identifying markers linked to the traits through constructing the DNA pools with extreme traits, and first used in identifying the resistance loci in lettuce (Michelmore et al., 1991). With the development of next generation sequencing (NGS) technologies, the high throughput SNPs and InDels can be rapidly detected from the genome through sequencing. Recently, a series of NGS based BSA+ approaches have been developed, including BSA-seq (or QTL-seq), BSR-seq, MutMup and MutMup+ (Abe et al., 2012, Fekih et al., 2013, Steuernagel et al., 2016, Takagi et al., 2013). In comparison to the food crops, such as rice and wheat, the reproduction coefficient of peanut is very low. It is difficult to fine mapping the genes and QTLs through traditional mapping methods. For BSA-seq, huge segregation population is not required, and it has been successfully used in peanut for identification of the candidate interval and development linked molecular marker for many traits, such as disease resistance, shelling percentage, dormancy, black testa and red testa (Clevenger et al., 2018a, Luo et al., 2018, Pandey et al., 2017, Zhao et al., 2020, Chen et al., 2021). In this study, the candidate interval associated with the red testa was identified through the BSA-seq method. Then, the traditional mapping method was used to construct the genetic linkage map and finally narrow down to the candidate gene in a 530-kbp interval. The strategy of combined BSA-seq and traditional mapping approach were used to accelerate the identification of the candidate gene AhRt2, which could be used to identify other genes in peanut and other crops.

\section{AhRt1 and AhRt2 were mapped in homologous fragment in different chromosomes}

Previous studies have suggested that at least three pairs of genes were responsible for the red testa of peanut including one dominant gene and two recessive genes (Branch, 2011). Recently, AhRt1, a major dominant gene controlling red testa, was identified in a 580-kb interval of chromosome A03 (Chr.03). One of the bHLH transcriptional factors was suggested as the possible candidate gene for AhRt1 (Chen et al., 2021). In the present study, a recessive gene controlling red testa color was successfully fine mapped to a physical interval of $530 \mathrm{~kb}$ of $\mathrm{Chr} .12$, and named as AhRt2. Functional annotation and sequence analysis suggested that AhRt2 could be the anthocyanidin reductase (ANR) coding gene.

Cultivated peanut is an allotetraploid with AA and BB subgenomes, and Chr.12 (B02) is corresponding to Chr.02 (A02). However, sequence alignment showed that there is no allele of Arahy.IK60LM in Chr.02. Instead, Arahy.IK60LM was mapped with the ANR gene Arahy.W8TDEC of Chr.03 with identity of $97.38 \%$. Interestingly, the physical distance between Arahy.W8TDEC and Arahy.07ZIFT (AhRt1) is only $262 \mathrm{~Kb}$. Further sequence alignment showed that most of the genes in the candidate interval (Chr12: $117.03 \mathrm{Mb}$ $117.56 \mathrm{Mb}$ ) could be mapped in Chr.03, suggesting that there is a homologous chromosome fragment between Chr.03 and Chr.12. So, it is interesting that AhRt1 and AhRt2 were mapped in homologous fragment of different sub genome of peanut. In addition, that chromosome fragment could be found between Chr.A03 of A. duranensis and Chr.B02 of A. ipaensis, implying that the chromosome fragment exchange might have occurred before the polyploidization of cultivated peanut.

\section{Detection of the SNPs of candidate gene in different peanut germplasm resources}


To further analyze the function of the candidate gene, we detected the two SNPs of Arahy.IK60LM in peanut germplasm resources with different testa colors, including five pink testa, eight red testa, one white testa, two black and two black stripe peanuts. For the SNP at the upstream of the CDS, most of the germplasms are with the same genotype as the red parent, only two peanuts with black stripe with the same genotype as YZ9102. For the SNP in the third exon of the candidate gene, the "G to A" was specific only in two red parents $\mathrm{ZH} 12$ and $\mathrm{ZH} 2$ (Figure 5). It implied that there was more than one gene controlling the red testa of cultivated peanut. The molecular mechanism of the other six red testa varieties was different from that of the $\mathrm{ZH} 12$ and $\mathrm{ZH} 2$.

\section{Development of diagnostic marker for screening the peanut with red testa}

We developed a KASP marker KASP_AhRt2 according to the sequence alignment in the candidate interval. A total of 220 lines of $Y Z Z H 12$ populations and 57 lines of $Y Z Z H 2$ populations were used for this site detection, and found that the locus of almost all red testa lines was " $\mathrm{T}: \mathrm{T}$ ", and the pink testa lines were "C:C" or " $\mathrm{T}: \mathrm{C}$ ", except YZZH12-127 and YZZH12-181(Figure 6). The two lines had red phenotype with "T:C" genotype. We found that the "SNP_117190528" of the two lines was consistent with the red parent by sequencing PCR products. Therefore, the two lines should be the linkage exchange between the KASP_AhRt2 and SNP_117190528. In addition, our results suggested that the KASP marker can be used as the diagnostic genotyping marker to predict red testa peanut through MAS. However, the diagnostic marker is only used in the cross event using the ZH12 or ZH2 as donor parent for the red testa needs further validation in different genetic backgrounds.

\section{Conclusion}

In our study, one gene regulating red testa of cultivated peanut, AhRt2, was mapped in a $530 \mathrm{~kb}$ region on chromosome 12 using BSA-seq and linkage mapping approaches. Both functional annotation and sequence analysis suggested that it is a anthocyanin reductase gene. In addition, a diagnostic marker (KASP_AhRt2) was developed for MAS in peanut. This work lays the foundation for the further understanding of the regulation mechanisms of peanut red testa formation and molecular breeding of new varieties with red testa.

\section{Declarations}

\section{Acknowledgements}

This research is supported by National Natural Science Foundation of China (32072090, 31861143009, 31801301), Key Research and Development Project of Shandong Province (2020LZGC001), Famous subjects construction project of Shandong Agricultural and Engineering University, High-level Foreign Experts Introduction Program (GL20200123001), and Taishan Scholar Project of Shandong Province (ts20190964). 
Availability of data and materials All information is specified in the manuscript or included as Additional Files.

Conflict of interest The authors declare that they have no conflict of interest.

\section{Authors' contribution statement}

Chuanzhi Zhao and Xingjun Wang conceived and designed the experiments. Mei Yuan, Jing Ma, Mingxiao Wang, Huiling Zhao and Jiaowen Pan developed the populations. Kun Zhang, Han Xia, Liangqiong He, Lei Hou, Shuzhen Zhao, Pengcheng Li, Ruizheng Tian, Guanghui Li, Mahendar Thudi and Changle Ma performed the experiments. Chuanzhi Zhao and Kun Zhang wrote the manuscript. Xingjun Wang revised the manuscript.

\section{References}

1. Abe A, Kosugi S, Yoshida K, et al., 2012. Genome sequencing reveals agronomically important loci in rice using MutMap. Nat Biotechno/30, 174-8.

2. Agarwal G, Clevenger J, Pandey MK, et al., 2018. High-density genetic map using whole-genome resequencing for fine mapping and candidate gene discovery for disease resistance in peanut. Plant Biotechnol J 16, 1954-67.

3. Bertioli DJ, Cannon SB, Froenicke L, et al., 2016. The genome sequences of Arachis duranensis and Arachis ipaensis, the diploid ancestors of cultivated peanut. Nat Genet 48, 438-46.

4. Bertioli DJ, Jenkins J, Clevenger J, et al., 2019. The genome sequence of segmental allotetraploid peanut Arachis hypogaea. Nat Genet 51, 877-84.

5. Branch W, 2011. First 100 years-inheritance of testa color in peanut (Arachis hypogaea L.). Crop Science 51, 1-4.

6. Chen H, Chen X, Xu R, et al., 2021. Fine-mapping and gene candidate analysis for AhRt1, a major dominant locus responsible for testa color in cultivated peanut. Theor Appl Genet 134(11):37213730 .

7. Chen X, Lu Q, Liu H, et al., 2019. Sequencing of cultivated peanut, Arachis hypogaea, yields insights into genome evolution and iil improvement. Mol Plant.

8. Chen Y, Chen Y, Shi C, et al., 2018. SOAPnuke: a MapReduce acceleration-supported software for integrated quality control and preprocessing of high-throughput sequencing data. Gigascience 7, 1-6.

9. Clevenger J, Chu Y, Chavarro C, et al., 2017. Genome-wide SNP genotyping resolves signatures of selection and tetrasomic recombination in peanut. Mol Plant 10, 309-22.

10. Clevenger J, Chu Y, Chavarro C, et al., 2018a. Mapping late leaf spot resistance in peanut (Arachis hypogaea) using QTL-seq reveals markers for marker-assisted selection. Front in Plant Sci 9, 83.

11. Clevenger JP, Korani W, Ozias-Akins P, Jackson S, 2018b. Haplotype-based genotyping in polyploids. Front Plant Sci 9, 564. 
12. Fekih $\mathrm{R}$, Takagi $\mathrm{H}$, Tamiru M, et al., 2013. MutMap+: genetic mapping and mutant identification without crossing in rice. PLoS One 8, e68529.

13. Giordano D, Locatelli M, Travaglia F, et al., 2017. Bioactive compound and antioxidant activity distribution in roller-milled and pearled fractions of conventional and pigmented wheat varieties. Food Chem 233, 483-91.

14. Han S, Yuan M, Clevenger JP, et al., 2018. A SNP-based linkage map revealed QTLs for resistance to early and late leaf spot diseases in peanut (Arachis hypogaea L.). Front Plant Sci 9, 1012.

15. Hill JT, Demarest BL, Bisgrove BW, Gorsi B, Su YC, Yost HJ, 2013. MMAPPR: mutation mapping analysis pipeline for pooled RNA-seq. Genome Res 23, 687-97.

16. Huang L, Liu X, Pandey MK, et al., 2020. Genome-wide expression quantitative trait locus analysis in a recombinant inbred line population for trait dissection in peanut. Plant Biotechnol J 18, 779-90.

17. Ito VC, Lacerda LG, 2019. Black rice (Oryza sativa L.): A review of its historical aspects, chemical composition, nutritional and functional properties, and applications and processing technologies. Food Chem 301, 125304.

18. Lei L, Zheng H, Bi Y, et al., 2020. Identification of a Major QTL and Candidate Gene Analysis of Salt Tolerance at the Bud Burst Stage in Rice (Oryza sativa L.) Using QTL-Seq and RNA-Seq. Rice ( $N$ Y) 13,55 .

19. Li H, Durbin R, 2009. Fast and accurate short read alignment with Burrows-Wheeler transform. Bioinformatics 25, 1754-60.

20. Li H, Qiu J, Chen X, Hong Y, Liang X, 2017. Relationship between expression of anthocyanin synthesis related genes and seed coat color in peanut. Chinese JOil Crops Sciences (Printed in chinese) 39, 600-5.

21. Luo H, Pandey MK, Khan AW, et al., 2018. Discovery of genomic regions and candidate genes controlling shelling percentage using QTL-seq approach in cultivated peanut (Arachis hypogaea L.). Plant Biotechnol J 17(7),1248-1260.

22. Ma J, Zhao Y, Chen $\mathrm{H}$, et al., 2020. Genome-wide development of polymorphic microsatellite markers and their application in peanut breeding program. Electronic $J$ Biotechno/ 4, 25-32.

23. Mancinelli AL, Rossi F, Moroni A, 1991. Cryptochrome, phytochrome, and anthocyanin production. Plant Physiol 96, 1079-85.

24. Mckenna A, Hanna M, Banks E, et al., 2010. The Genome Analysis Toolkit: a MapReduce framework for analyzing next-generation DNA sequencing data. Genome Res 20, 1297-303.

25. Meng L, Li H, Zhang L, Wang JJTCJ, 2015. QTL IciMapping: integrated software for genetic linkage map construction and quantitative trait locus mapping in biparental populations. The Crop Journa/3, 269-83.

26. Michelmore RW, Paran I, Kesseli R, 1991. Identification of markers linked to disease-resistance genes by bulked segregant analysis: a rapid method to detect markers in specific genomic regions by using segregating populations. ProcNat AcadSci USA 88, 9828-32. 
27. Nabi RBS, Cho KS, Tayade R, et al., 2021. Genetic diversity analysis of Korean peanut germplasm using 48 K SNPs 'Axiom_Arachis' Array and its application for cultivar differentiation. Sci Rep 11, 16630.

28. Pandey MK, Khan AW, Singh VK, et al., 2017. QTL-seq approach identified genomic regions and diagnostic markers for rust and late leaf spot resistance in groundnut (i L.). Plant Biotechnol J 15, 927-41.

29. Pandey MK, Monyo E, Ozias-Akins $P$, et al., 2012. Advances in Arachis genomics for peanut improvement. Biotechnol Adv 30, 639-51.

30. Shin WH, Park SJ, Kim EJ, 2006. Protective effect of anthocyanins in middle cerebral artery occlusion and reperfusion model of cerebral ischemia in rats. Life Sci 79, 130-7.

31. Steuernagel B, Periyannan SK, Hernandez-Pinzon I, et al., 2016. Rapid cloning of disease-resistance genes in plants using mutagenesis and sequence capture. Nat Biotechno/ 34, 652-5.

32. Takagi H, Abe A, Yoshida K, et al., 2013. QTL-seq: rapid mapping of quantitative trait loci in rice by whole genome resequencing of DNA from two bulked populations. Plant $J \mathbf{7 4}, 174-83$.

33. Teng S, Keurentjes J, Bentsink L, Koornneef M, Smeekens S, 2005. Sucrose-specific induction of anthocyanin biosynthesis in Arabidopsis requires the MYB75/PAP1 gene. Plant Physio/ 139, 184052.

34. Voorrips RE, 2002. MapChart: software for the graphical presentation of linkage maps and QTLs. $J$ Hered $93,77-8$.

35. Wang J, Zhang Q, Cui F, et al., 2017. Genome-wide analysis of gene expression provides new insights into cold responses in Thellungiella salsuginea. Frontiers in Plant Science 8, 713.

36. Winkel-Shirley B, 2001. Flavonoid biosynthesis. A colorful model for genetics, biochemistry, cell biology, and biotechnology. Plant Physiol 126, 485-93.

37. Xue Q, Zhang $X$, Yang H, et al., 2021. Transcriptome and metabolome analysis unveil anthocyanin metabolism in pink and red testa of peanut (Arachis hypogaea L.). Int J Genomics 5883901.

38. Yin D, Ji C, Ma X, et al., 2018. Genome of an allotetraploid wild peanut Arachis monticola: a de novo assembly. Gigascience 7.

39. Zhao C, Qiu J, Agarwal G, et al., 2017. Genome-Wide discovery of microsatellite markers from diploid progenitor species, Arachis duranensis and A. ipaensis, and their application in cultivated peanut ( $A$. hypogaea). Front Plant Sci 8, 1209.

40. Zhao X, Chen J, Du F, 2012. Potential use of peanut by-products in food processing: a review. J Food Science Technology 49, 521-9.

41. Zhao Y, Ma J, Li M, et al., 2020. Whole-genome resequencing-based QTL-seq identified AhTc1 gene encoding a R2R3-MYB transcription factor controlling peanut purple testa colour. Plant Biotechnol J 18, 96-105.

42. Zhuang $\mathrm{W}$, Chen $\mathrm{H}$, Yang $\mathrm{M}$, et al., 2019. The genome of cultivated peanut provides insight into legume karyotypes, polyploid evolution and crop domestication. Nat Genet 51, 865-76. 


\section{Tables}

Table 1 Genetic analysis of red testa colour in peanut

Name of the population Generation Total plantsPinkRedExpected ratio $\chi^{2} P$-value

\begin{tabular}{|c|c|c|c|c|c|c|c|c|c|c|}
\hline \multirow{2}{*}{\multicolumn{3}{|c|}{$\begin{array}{c}\text { YZZH12 } \\
\text { वYZ9102× ZhongHua } 12 \text { } \\
\text { YZZH2 } \\
\text { वYZ9102× Zhanhong } 2 \square\end{array}$}} & \multirow{2}{*}{$\begin{array}{l}\mathrm{F}_{4} \\
\mathrm{~F}_{4}\end{array}$} & \multirow{2}{*}{$\begin{array}{l}220 \\
271\end{array}$} & \multirow{2}{*}{$\begin{array}{ll}143 & 77 \\
176 & 95\end{array}$} & \multirow{2}{*}{$\begin{array}{l}5: 3 \\
5: 3\end{array}$} & \multirow{2}{*}{$\begin{array}{l}0.59 \\
0.69\end{array}$} & \multirow{2}{*}{$\begin{array}{l}0.05 \\
0.05\end{array}$} & \multicolumn{2}{|c|}{$\chi^{2} \square 3.85$} \\
\hline & & & & & & & & & \multirow{2}{*}{\multicolumn{2}{|c|}{$\begin{array}{l}\text { Table } 2 \text { Data } \\
\text { generated by } \\
\text { BSA-seq }\end{array}$}} \\
\hline \multirow[b]{2}{*}{ Samples } & \multirow[b]{2}{*}{ Clean reads } & \multirow{2}{*}{\multicolumn{2}{|c|}{ Clean bases }} & \multirow{2}{*}{\multicolumn{2}{|c|}{$\begin{array}{l}\text { Number of reads } \\
\text { Mapped }\end{array}$}} & \multirow[b]{2}{*}{$\begin{array}{l}\text { Mapping } \\
\text { ratio (\%) }\end{array}$} & \multirow[b]{2}{*}{$\begin{array}{l}\text { Depth } \\
\text { (x) }\end{array}$} & \multirow{2}{*}{\multicolumn{2}{|c|}{$\begin{array}{l}\qquad \text { BS } \\
\text { Properly } \\
\text { paired read }\end{array}$}} & \\
\hline & & & & & & & & & & $\begin{array}{l}\text { Properly } \\
\text { paired ratio }\end{array}$ \\
\hline YZ9102 & $210,495,768$ & $31,5^{\prime}$ & 365,200 & 210,63 & 335 & 99.97 & 11.28 & 209,08 &, 834 & $99.33 \%$ \\
\hline ZH12 & $274,833,602$ & 41,2 & 040,300 & 275,07 & 939 & 99.99 & 14.72 & 272,5 &, 740 & $99.17 \%$ \\
\hline $\begin{array}{l}\text { Pink- } \\
\text { pool }\end{array}$ & $755,040,492$ & 113, & $, 073,800$ & 803,08 & 248 & 99.95 & 40.45 & 795,20 & 1,596 & $99.08 \%$ \\
\hline $\begin{array}{l}\text { Red- } \\
\text { pool }\end{array}$ & $802,571,036$ & 120 & $5,655,400$ & 754,68 & 636 & 99.83 & 42.99 & 747,42 & 1,150 & $98.99 \%$ \\
\hline
\end{tabular}

Table 3 Gene information in the candidate region on $\mathrm{Chr} .12$ 


\begin{tabular}{|c|c|c|c|}
\hline Gene & Start & End & Function \\
\hline Arahy.1KM4NQ & 117005569 & 117007230 & MYB transcription factor MYB48 \\
\hline Arahy.IZM7T4 & 117019886 & 117024789 & Protein kinase superfamily protein \\
\hline Arahy.BL8W3W & 117025116 & 117031218 & Transcription factor jumonji (jmjC) domain-containing protein \\
\hline Arahy.VDG15V & 117045486 & 117047405 & Octicosapeptide/Phox/Bem1p family protein \\
\hline Arahy.B3SD 72 & 117048799 & 117051828 & Unknown protein \\
\hline Arahy.J2UCUC & 117052969 & 117055610 & Cellulase (glycosyl hydrolase family 5) \\
\hline Arahy.6S25R2 & 117069253 & 117081682 & receptor-like serine/threonine kinase \\
\hline Arahy.WE5WNX & 117081781 & 117085905 & receptor-like serine/threonine kinase \\
\hline Arahy.KK3MU5 & 117089125 & 117096591 & Unknown protein \\
\hline Arahy.D43PH9 & 117097423 & 117101091 & receptor-like serine/threonine kinase \\
\hline Arahy.4HL1MH & 117115390 & 117118208 & Quinone reductase family protein \\
\hline Arahy.X3FWT9 & 117131960 & 117135240 & transcription factor bHLH123-like \\
\hline Arahy.DOFPDW & 117169647 & 117170588 & transcription factor DYSFUNCTIONAL TAPETUM 1-like \\
\hline Arahy.EYN48E & 117173896 & 117177659 & terpene synthase \\
\hline Arahy.7M140W & 117182257 & 117188503 & FYVE zinc finger protein \\
\hline Arahy.IK60LM & 117188946 & 117191576 & anthocyanidin reductase ((2S)-flavan-3-ol-forming \\
\hline Arahy.ME5340 & 117192337 & 117194676 & Unknown protein \\
\hline Arahy.XPM13Z & 117195285 & 117195741 & Unknown protein \\
\hline Arahy. $6345 F V$ & 117195818 & 117200790 & (2R)-phospho-3-sulfolactate synthase ComA \\
\hline Arahy.R4UZW9 & 117212854 & 117218115 & ATP binding microtubule motor family protein \\
\hline Arahy.0BBN6W & 117218341 & 117220729 & Sulfite exporter TauE/SafE family protein \\
\hline Arahy.92LHRM & 117231502 & 117234533 & receptor-like kinase 1 \\
\hline Arahy.77GZB8 & 117272366 & 117277939 & branched-chain amino acid transaminase 2 \\
\hline Arahy.UIBCOV & 117280589 & 117283465 & branched-chain amino acid transaminase 2 \\
\hline Arahy.BHC7EV & 117284545 & 117287751 & Unknown protein \\
\hline Arahy.FCW1MU & 117311541 & 117316007 & $\begin{array}{l}\text { CBS domain-containing protein with a domain of unknown function } \\
\text { (DUF21) }\end{array}$ \\
\hline Arahy.K6XKIL & 117316426 & 117318345 & Unknown protein \\
\hline Arahy.FI0236 & 117318361 & 117320009 & Unknown protein \\
\hline Arahy.VXEK44 & 117322449 & 117330848 & LL-diaminopimelate aminotransferase \\
\hline Arahy.3DBGOK & 117343268 & 117346310 & malate dehydrogenase \\
\hline Arahy.JFV18T & 117345716 & 117349561 & basic helix-loop-helix (bHLH) DNA-binding superfamily protein \\
\hline Arahy.WHNG6D & 117365459 & 117375941 & DHHC-type zinc finger family protein \\
\hline Arahy.UW1SNI & 117376910 & 117380446 & inorganic pyrophosphatase \\
\hline Arahy.T1IW9N & 117377016 & 117379835 & Unknown protein \\
\hline Arahy.WTJOL8 & 117380766 & 117380972 & Unknown protein \\
\hline Arahy.JM8NV3 & 117382006 & 117386697 & Major facilitator superfamily protein \\
\hline Arahy.7CPH5R & 117414427 & 117417456 & Glutamyl-tRNA reductase family protein \\
\hline Arahy.Z3ANCQ & 117417878 & 117423386 & Unknown protein \\
\hline Arahy.52ZQRU & 117419135 & 117420277 & LisH/CRA/RING-U-box domains-containing protein \\
\hline Arahy.5N4DPF & 117423355 & 117426869 & Unknown protein \\
\hline Arahy.TBP304 & 117428488 & 117430556 & ubiquitin carboxyl-terminal hydrolase-like protein \\
\hline Arahy.LX50IY & 117442385 & 117450867 & LRR and NB-ARC domain disease resistance protein \\
\hline Arahy.YEAJ9J & 117460320 & 117470837 & LRR and NB-ARC domain disease resistance protein \\
\hline Arahy.R7V7OE & 117482548 & 117487344 & LRR and NB-ARC domain disease resistance protein \\
\hline Arahy.FTDU9L & 117483618 & 117494316 & Unknown protein \\
\hline Arahy.U1EPQF & 117490366 & 117494234 & LRR and NB-ARC domain disease resistance protein \\
\hline Arahy. $Y 1 X B L J$ & 117495399 & 117498854 & Unknown protein \\
\hline Arahy.1L886L & 117501455 & 117505674 & disease resistance protein (TIR-NBS-LRR class) \\
\hline Arahy.P56WT2 & 117510793 & 117512637 & Unknown protein \\
\hline
\end{tabular}


Table 4 SNPs in putative candidate genes in the mapping genomic region for testa color on Chr.12

\begin{tabular}{llllllll}
\hline SNP Position & $\Delta$ SNP & ED & Ref & Alt & Al & Genes & Gene annotation \\
\hline 117049317 & 0.94 & 1.33 & C & G & Exon & Arahy.B3SD72 & Uncharacterized protein \\
117191491 & - & 1.28 & G & A & 5'UTR & Arahy.IK60LM & Anthocyanidin reductase \\
\hline 117190528 & 0.95 & 1.35 & G & A & Exon & Arahy.IK60LM & Anthocyanidin reductase \\
\hline 117328756 & 0.89 & 1.27 & A & G & 3'UTR & Arahy.VXEK44 & Diaminopimelate aminotransferase \\
\hline 117420658 & 0.92 & 1.29 & A & G & 3'UTR & Arahy.Z3ANCQ & Protein trichome birefringence-like \\
\hline 117444731 & 0.9 & 1.27 & A & G & Intron & Arahy.LX50IY & NBS-LRR disease resistance protein \\
\hline 117444733 & 0.9 & 1.27 & A & C & Intron & Arahy.LX50IY & NBS-LRR disease resistance protein \\
\hline 117504480 & 0.92 & 1.30 & T & C & Exon & Arahy.1L886L & NBS-LRR disease resistance protein \\
\hline
\end{tabular}

"Ref" is the SNP genotype in the reference genome, while the "Alt" is the genotype in the non-reference genome. “Al” indicates the functional location of identified SNP in annotated candidate gene.

\section{Figures}

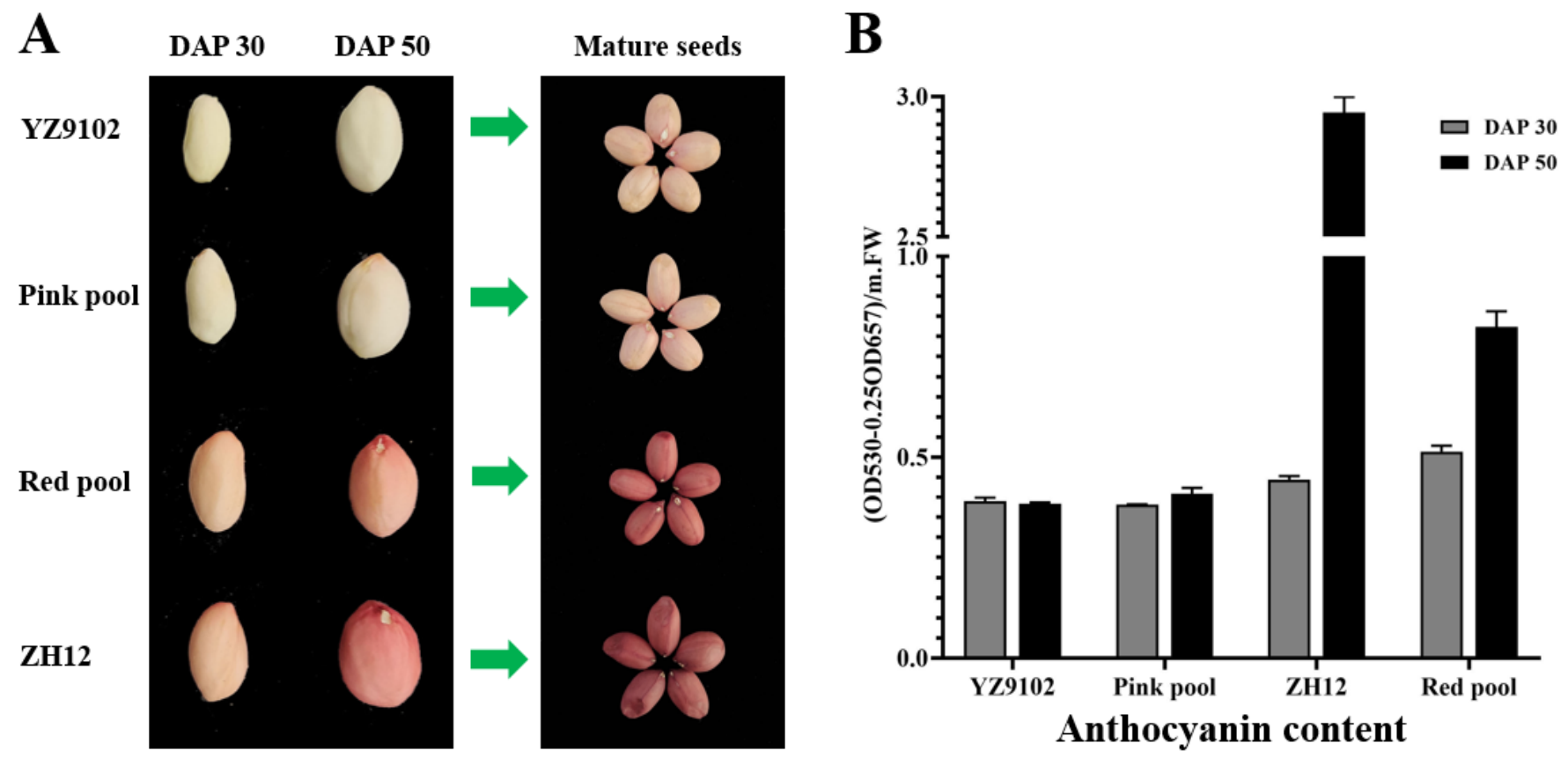

Figure 1 
Phenotypes and anthocyanin content (" DAP 30 " means 30 days after pegging; " DAP 50 " means 50 days after pegging ). a Seeds of pink parental line (YZ9102), red parental line (ZH12) and F4 lines with pink and red testa in different development stages, $b$ relative anthocyanins content in parental lines and homozygous F4 lines with testa color character.

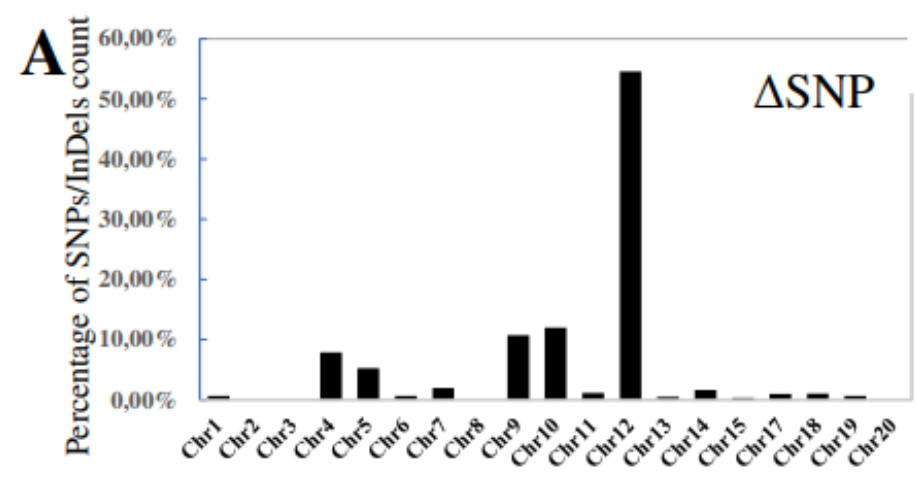

Chromosomes

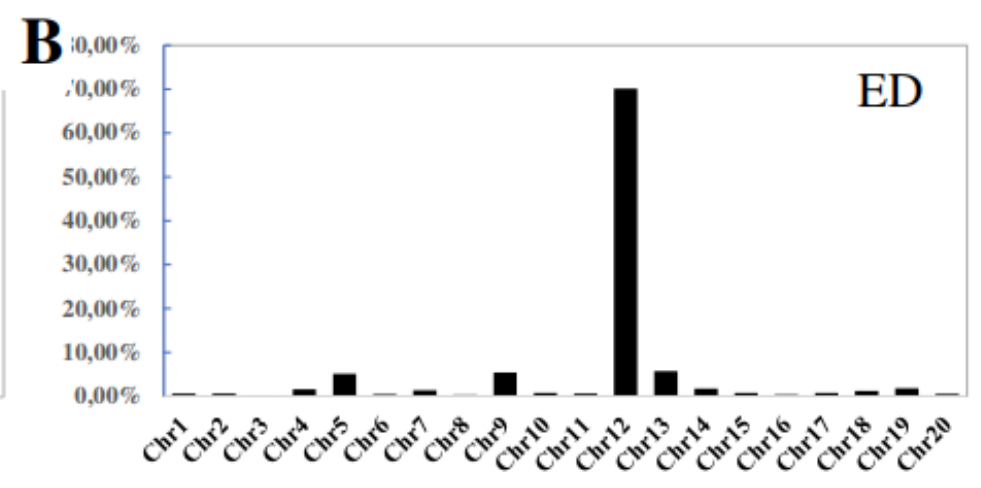

Chromosomes

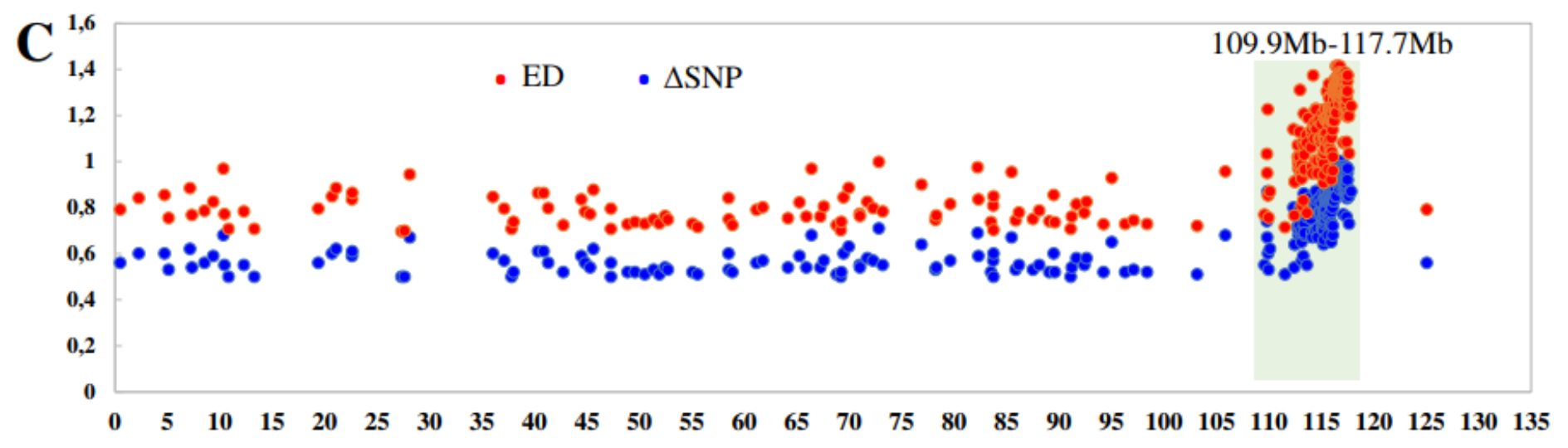

Figure 2

Distribution of candidate SNPs and InDels per chromosome. a Candidate SNPs and InDels using $\triangle S N P$ algorithm with a cutoff of $\triangle S N P>0.5$. b The top $1 \%$ SNPs and InDels using ED algorithm. $c$ Distribution of candidate SNPs/InDels on Chr.12. The significant region identified for red testa pheotype is shaded (109.9-117.7 Mb). 


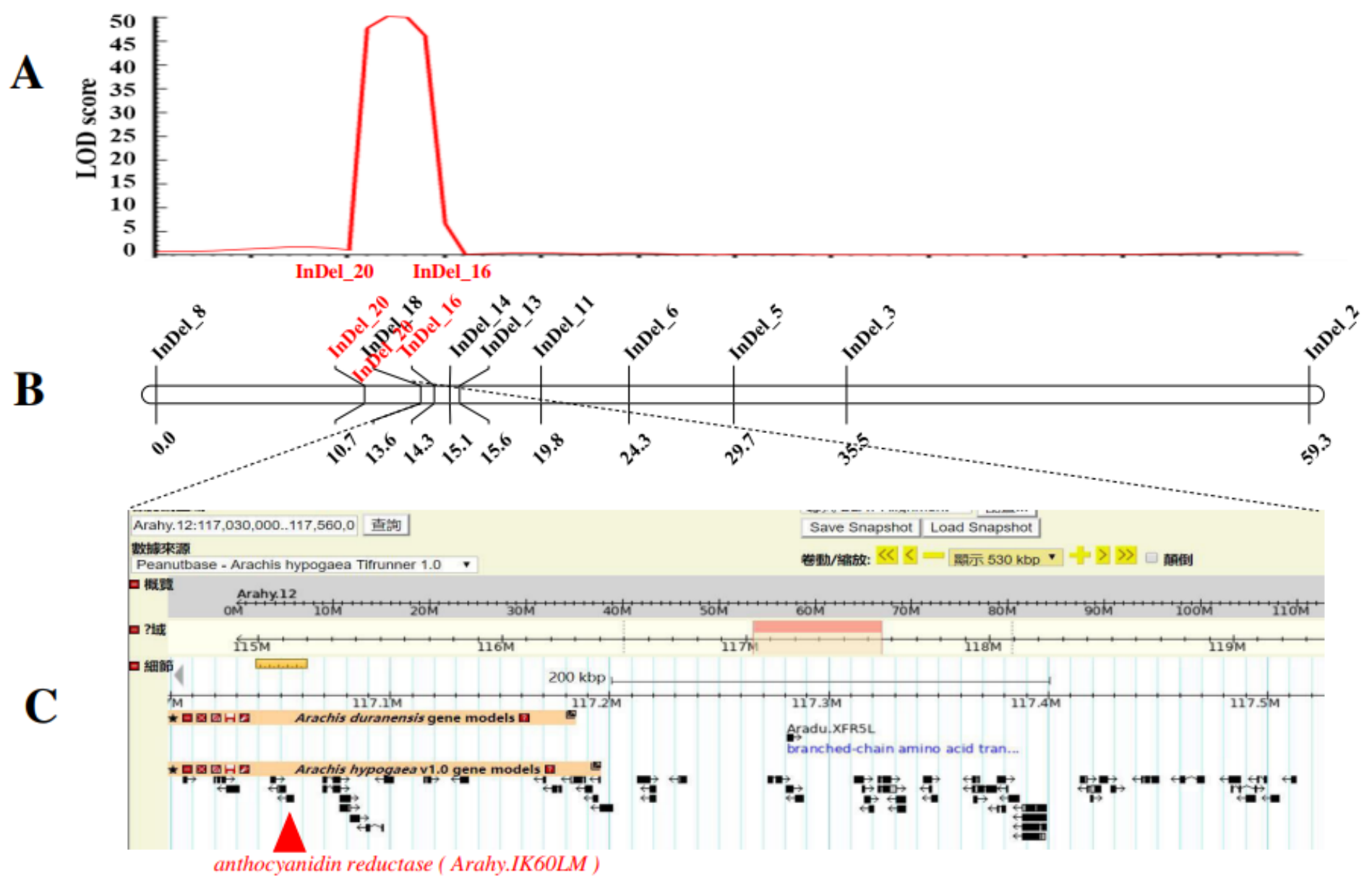

Chr. $12: 117.03-117.56 \mathrm{Mb}$

\section{Figure 3}

Identification of the candidate genes of AhRt2 through QTL mapping. a Narrowing the candidate region through IciMapping. The $x$-axis means the linkage groups corresponding to the candidate region of Chr.12 of peanut. The $y$-axis means the LOD scores. $b$ The genetic linkage map of candidate region of Chr.12. $\mathrm{c}$ the genes in the $0.5 \mathrm{Mb}$ interval. 
A

Arahy.IK60LM

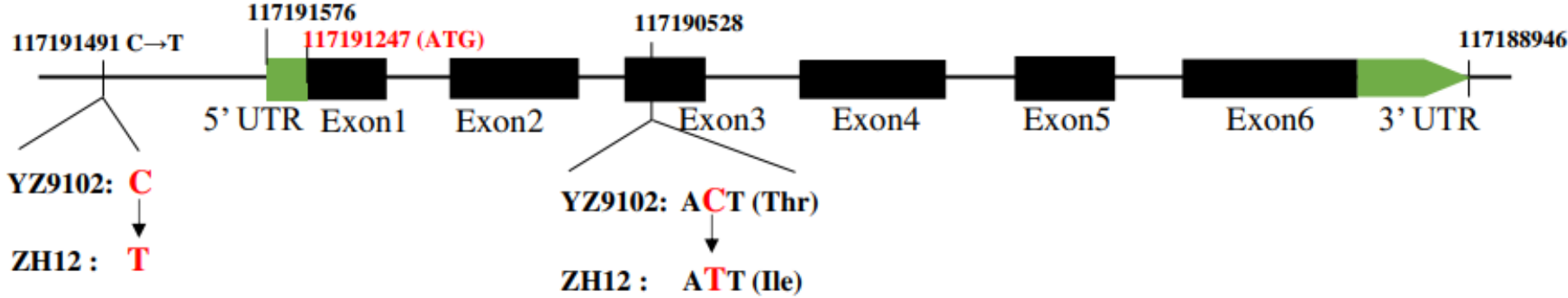

B
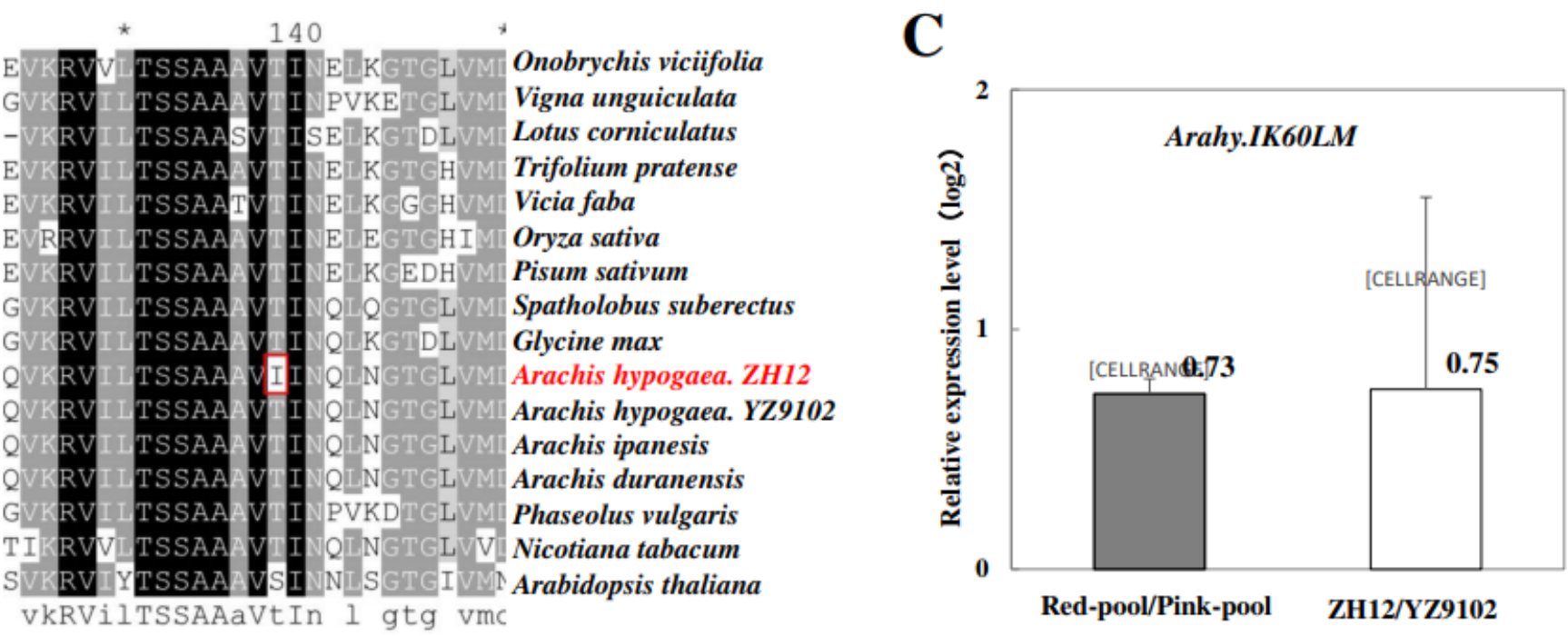

Figure 4

Gene structure and expression analysis of candidate gene. a Gene structure of candidate gene and the locations of the SNPs. b Sequence alignment of the ANR in different species. c Expression of the candidate gene in the parental lines and pools with the red and pink testa, respectively 
A

SNP: Ch12.117191491

(at -312 bp of AhRt2)

YZ9102 T C G G A T T

Huayu22 T C G A A $T$ T

Huayu23 T C G A A $T$ T

Yuhua14 T C G A A T T

Kaixuan016 T C G A A $\mathrm{T}$ T

Zhanhong2 $\mathrm{T}$ C G A A $\mathrm{T}$ T

Zhonghua $12 \mathrm{~T}$ C G A A $\mathrm{T}$ T

DBSLH T C G A A $T$ T

Chizhenzhu T C G A A $T$ T

LNSLH T C G A A $\mathrm{T}$ T

Guihuahong166 T C G A A T T

Zhenzhuhong1 T C G A A T T

PI356004 T C G A A $\mathrm{T}$ T

Kainongbai T C G A A T T white testa

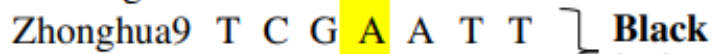

Heihuasheng $\mathrm{T}$ C G A A T T $\}$ testa

PI502120 T C G G A $T$ T $T$ C Black

Qicai T C G G A T T $\}$ stripe

\section{B $\quad \begin{gathered}\text { SNP: Ch12.117190528 } \\ \text { (at the third exon of AhRt2) }\end{gathered}$}

$\left.\begin{array}{lllllllll}\text { YZ9102 A } & T & A & G & T & C & A\end{array}\right]$

$\begin{array}{llllllllll}\text { Huayu23 A } & \text { T } & \text { A } & \text { G } & \text { T } & \text { C } & \text { A } & \text { Pink }\end{array}$

Yuhua14 A $T$ A $G$ G C A $\quad$ testa

$\begin{array}{llllllll}\text { Kaixuan016 A } & \text { T } & \text { A } & \text { G } & \text { T } & \text { C } & \text { A }\end{array}$

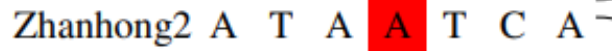

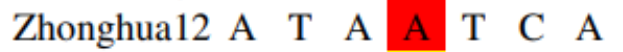

$\begin{array}{llllllll}\text { DBSLH A } & T & A & G & T & C & A\end{array}$

Chizhenzhu A T A A

LNSLH A $T$ T A

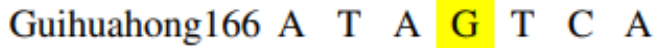

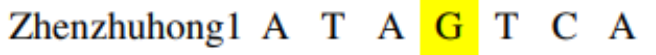

$\begin{array}{llllllll}\text { PI356004 A } & \text { T } & \text { A } & G & T & C & A\end{array}$

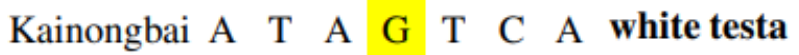

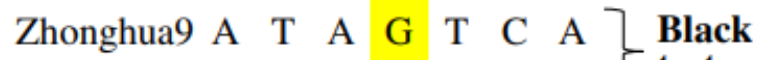

Heizhenzhu A T A G T C A $\}$ testa

$\begin{array}{llllllll}\text { PI502120 A } & \text { T } & \text { A } & G & T & C & A\end{array}$ Black

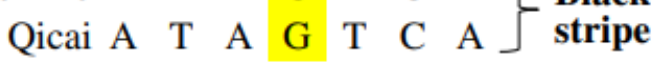

\section{Figure 5}

Detecting of SNPs of candidate gene of AhRt2 in different peanut germplasms. a Detecting of SNPs at the -312 bp of the initiation codon of the candidate gene. b Detecting of SNPs at the third exon of AhRt2.

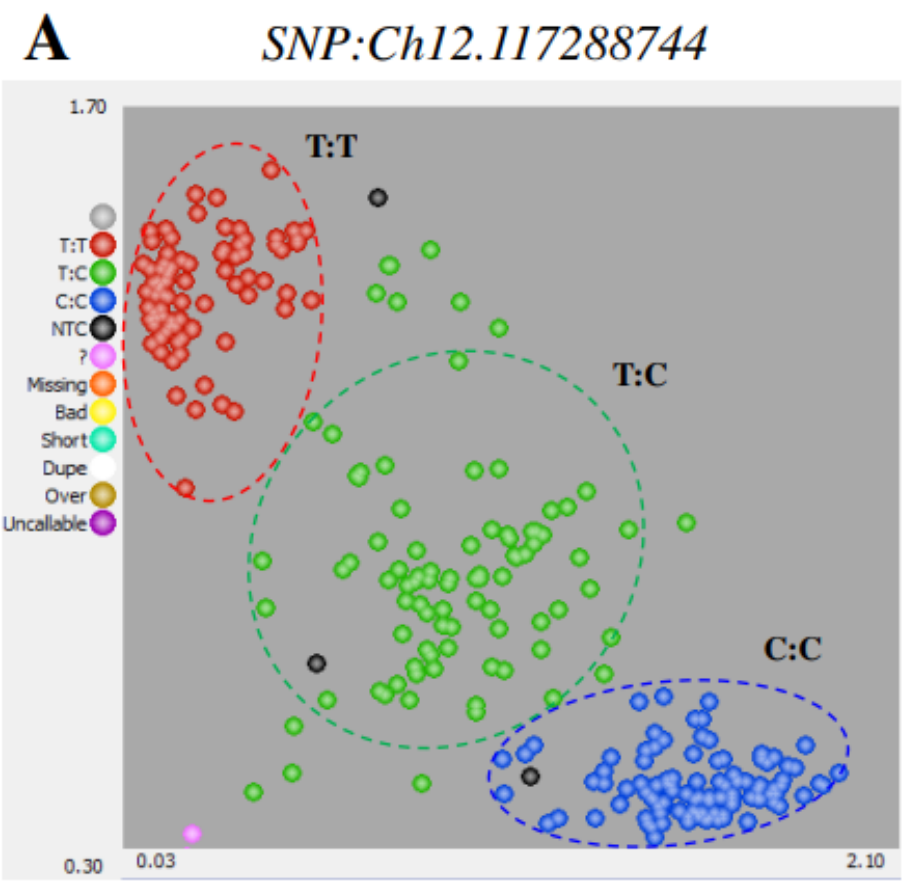

B

YZZH12-3: TATATATTT[T/T]TTCTTTATT

YZZH12-4: TATATATTT[T/T]TTCTTTATT

YZZH12-127: TATATATTT[T/C]TTCTTTATT

YZZH12-181: TATATATTT[T/C]TTCTTTATT

YZZH12-1: TATATATTT[C/C]TTCTTTATT

YZZH12-2: TATATATTT[T/C]TTCTTTATT

..... $220 \mathrm{YZZH} 12 \mathrm{~F}_{4}$ lines

YZZH2-1: TATATATTT[T/T]TTCTTTATT

YZZH2-2: TATATATTT[T/T]TTCTTTATT

YZZH2-3: TATATATTT[C/C]TTCTTTATT

YZZH2-4: TATATATTT[C/C $]$ TTCTTTATT

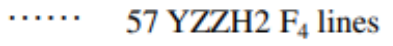


Figure 6

Genotyping results of SNP:Ch12.117288744 by KASP(Kompetitive Allele Specific PCR).a The scatter plot with axes $\mathrm{x}$ and $\mathrm{y}$ represents allelic discrimination of this site genotypes. The red, green and blue dots represent the mutant homozygous, heterozygous and wild-type homozygous, respectively. b Validation of diagnostic marker in YZZH12 and YZZH2 populations.

\section{Supplementary Files}

This is a list of supplementary files associated with this preprint. Click to download.

- TableS1Markersandprimersusedinthisstudy.xlsx 